\title{
Masses of charm and strange baryons
}

\author{
James Bjorken* \\ Fermilab
}

(Dated: Aug 13, 1986)

\section{INTRODUCTION}

Our intent is to make simple-minded mass estimates of the baryons containing charm and strange quarks. We use the following strategy:

1. Assume $(c c c) / \psi$ and $(b b b) / \Upsilon$ mass ratios are obtainable from a reasonable extrapolation of $\Delta^{++} / \rho, \omega$ and $\Omega^{-} / \phi$.

2. Assume the equal-spacing rule for masses within the $J=\frac{3}{2}$ multiplet to

3. Estimate hyperfine splittings for $J=\frac{1}{2}$ baryons via extrapolation of trends from existing data.

\section{MASSES OF $\Omega_{c c c}^{++}$AND $\Omega_{b b b}^{-}$}

We have

$$
\begin{gathered}
\frac{\Delta^{++}}{\frac{1}{2}(\rho+\omega)}=\frac{1232}{776}=1.588 \\
\frac{\Omega^{-}}{\phi}=\frac{1672}{1020}=1.639
\end{gathered}
$$

It is reasonable to expect a tendency for decrease toward 1.50 as the mass scale increases. We take

$$
\frac{\Omega_{c c c}^{++}}{\psi}=1.60 \pm 0.02
$$

and

$$
\frac{\Omega_{b b b}^{-}}{\Upsilon}=1.57 \pm 0.02
$$

*Electronic address: bjorken@slac.stanford.edu 
leading to

$$
\begin{gathered}
\Omega_{c c c}^{++}=4955 \pm 62 \\
\Omega_{b b b}^{-}=14852 \pm 189
\end{gathered}
$$

An equal-spacing rule leads to the following mass formula

$$
m=\frac{1}{3}\left[1232\left(n_{u}+n_{d}\right)+1672 n_{s}+4955 n_{c}+14852 n_{b}\right] \pm 21 n_{c} \pm 63 n_{b}
$$

where we have added estimated errors for the uncertainty in the $c$ and $b$ mass scale. In Table I we tabulate the resultant $J=\frac{3}{2}$ masses for the relevant baryons. We add errors linearly, not in quadrative.

\section{HYPERFINE INTERVALS}

\section{A. Baryons with Two Identical Quarks}

In this case the identical quarks form a diquark of spin 1 which is color antitriplet. The spin of the third quark is either parallel $\left(J=\frac{3}{2}\right)$ or anti-parallel $\left(J=\frac{1}{2}\right)$ to the diquark. The magnitude of the splitting is in inverse proportion to the product of the masses of the like and unlike quarks. These we take to be $300 \mathrm{MeV}$ for $u$ and $d, 450 \mathrm{MeV}$ for $s, 1550 \mathrm{MeV}$ for $c$, and $4850 \mathrm{MeV}$ for $b$.

The actual hyperfine splittings also depend on dynamics, i.e. $|\psi(0)|^{2}$ for diquark wave functions. These effects are, out of ignorance, disregarded.

Table II exhibits the hyperfine splittings which we estimate here. Known values are indicated by an underline. Notice that the ratios of $p / \Sigma^{+}$hyperfine splittings is $294 / 194=1.51 \cong 450 / 300$ as assumed. Also, our model predicts the splitting for the $(q q) Q$ baryon equals that of the $(Q Q) q$. This is checked in the $\Xi^{0} / \Sigma^{+}$ratio $=216 / 194=1.11$, which is not too bad. Therefore we believe the error estimates to be fairly conservative.

Note that our notation is to label the $J=\frac{3}{2}$ baryon as $B^{*}$ and the $\Sigma$-like $J=\frac{1}{2}$ baryon as $B^{\prime}$. The $\Lambda$-like (ground-state) $J=\frac{1}{2}$ baryon will be labeled as $\mathrm{B}$.

\section{B. Baryons with No Identical Quarks}

Here there is no Pauli exclusion principle which applies and there are $8=2^{3}$ distinct states: 4 for the $J=\frac{3}{2} B^{*}$ and $2+2$ for the $J=\frac{1}{2} B^{\prime}$ and $J=\frac{1}{2} B$. If these are two light 
quarks and one heavy quark ( $c$ or $b$ ) we assign the light quarks to the diquark. If there are two heavy quarks and one light quark, we assign the heavies to the diquark. Table III lists the relevant states. There are two level spacings to deal with. Known values are underlined. Others which are obtained via isospin rotations from Table II are underlined with a dashed line.

Notice that the $B^{\prime}-B$ mass difference increases with increasing heavy-quark mass in $(u d) Q$ baryons. This occurs because we are changing the spins of the $u$ and $d$ from triplet to singlet in these systems; hence if there is no contribution from the third quark the splitting should be typical of splittings in the light meson systems (hundreds of $\mathrm{MeV}$ ).

Our assignment for $(u d) b$ reflects the trend seen (??) in the $\Sigma_{c}$ system. Note that in so

doing we assume that the $\Sigma_{c}$ seen is $\Sigma_{c}=\Sigma_{c}^{\prime}\left(J=\frac{1}{2}\right)$ and not $\Sigma_{c}^{*}$. Otherwise $\Sigma_{c}^{++}$itself would be stable under strong interactions. It would be a little surprising if a stable $\Sigma_{c}^{++}$ existed, as yet undiscovered.

The assignments for the $(u s) Q$ systems generalize the above considerations, with a $50 \%$ diminution because of the larger strange quark mass.

The estimates for $(b c) u$ (and similarly $(b c) s$ ) are, for the $B^{*}-B^{\prime}$ splitting, obtained by interpolation of the relevant $(b b) u$ and $(c c) u$ splittings, but the $B^{\prime}-B$ splittings are essentially guesses. It will take a while, however, before being proven wrong.

\section{BARYON MASSES AND THEIR IMPLICATIONS}

The masses of the ground state baryons are shown in the tables. Agreement with $\Lambda_{c}, A$, and $T$ are quite satisfactory. Note that $\Sigma_{c}$ is fitted, not predicted. We have not included the ISR value for $\Lambda_{b}$ mass of $5425_{-175}^{+175} \mathrm{MeV}$, since it is, to say the least, a controversial observation. But, if correct, it is evidently consistent with the estimate.

More relevant than absolute masses are the splittings. Most $B^{\prime}$ and $B^{*}$ states decay to the $B$ ground-state by pion emission. Interesting are the candidates for radiative decay. We list these in Table IV. The A, T, bss, ccu, ccd, and ccs are perhaps the best candidates, with the $\Xi_{b}$ (bus) excited states possibilities as well. 
TABLE I: Masses of $J=\frac{3}{2}$ Baryons (MeV)

\begin{tabular}{|c|c|}
\hline$\Omega_{b b b}^{-}(b b b)$ & $14852 \pm 189$ \\
\hline$\Omega_{b b c}^{0}(b b c)$ & $11553 \pm 147$ \\
\hline$\Omega_{b b}^{-}(b b s)$ & $10459 \pm 126$ \\
\hline$\Xi_{b b}^{0 *}(b b u)$ & $10312 \pm 126$ \\
\hline$\Omega_{b c c}^{+}(b c c)$ & $8254 \pm 105$ \\
\hline$\Omega_{b}^{-}(b s s)$ & $6065 \pm 63$ \\
\hline$\Sigma_{b}^{+*}(b u u)$ & $5772 \pm 63$ \\
\hline$\Omega_{b c}^{0}(b c s)$ & $7160 \pm 84$ \\
\hline$\Xi_{b c}^{+*}(b c u)$ & $7013 \pm 84$ \\
\hline$\Xi_{b}^{0 *}(b s u)$ & $5919 \pm 63$ \\
\hline$\Omega_{c c c}^{++}(c c c)$ & $4955 \pm 63$ \\
\hline$\Omega_{c c}^{+}(c c s)$ & $3861 \pm 42$ \\
\hline$\Xi_{c c}^{++*}(c c u)$ & $3714 \pm 42$ \\
\hline$\Omega_{c}^{0}(c s s)$ & $2766 \pm 21$ \\
\hline$\Sigma_{c}^{++*}($ cuu $)$ & $2473 \pm 21$ \\
\hline$\Xi_{c}^{+*}(c s u)$ & $2620 \pm 21$ \\
\hline
\end{tabular}


TABLE II: Hyperfine Splittings of Baryons Containing two Identical Quarks

\begin{tabular}{ccc}
\hline \hline & $B^{*}-B^{\prime}$ & $B^{\prime}$ mass \\
\hline$N^{+}(u u) d$ & $\underline{294}$ & 938 \\
$\Sigma^{+}(u u) s$ & $\underline{194}$ & 1189 \\
$\Sigma_{c}^{++}(u u) c$ & $56 \pm 20$ & $2417 \pm 41(\underline{2448})$ \\
$\Sigma_{b}^{+}(u u) b$ & $18 \pm 5$ & $5754 \pm 68$ \\
& & \\
$\Xi^{-}(s s) d$ & $\underline{216}$ & 1321 \\
$\Omega_{c}^{0}(s s) c$ & $42 \pm 15$ & $2724 \pm 36(\underline{2740} ?)$ \\
$\Omega_{b}^{-}(s s) b$ & $13 \pm 5$ & $6052 \pm 68$ \\
& & $3668 \pm 62$ \\
$\Xi_{c c}^{+}(c c) d$ & $56 \pm 20$ & $3819 \pm 57$ \\
$\Omega_{c c}^{+}(c c) s$ & $42 \pm 15$ & $8250 \pm 107$ \\
$\Omega_{c c b}^{+}(c c) b$ & $4 \pm 2$ & $10294 \pm 131$ \\
$\Xi_{b b}^{-}(b b) d$ & $18 \pm 5$ & $10446 \pm 131$ \\
$\Omega_{b b}^{-}(b b) s$ & $13 \pm 5$ & \\
$\Omega_{b b c}^{0}(b b) c$ & $4 \pm 2$ & \\
\hline & &
\end{tabular}

TABLE III: Hyperfine Splittings of Baryons Containing 3 Different Flavors of Quarks

\begin{tabular}{ccccc}
\hline \hline & $B^{*}-B^{\prime}$ & $B^{\prime}-B$ & $B^{\prime}$ mass & $B$ mass \\
\hline$\Sigma_{0}, \Lambda^{0}(u d) s$ & $\underline{192}$ & $\underline{77}$ & $\underline{1192}$ & $\underline{1116}$ \\
$\Sigma_{c}, \Lambda_{c}^{+}(u d) c$ & $56 \pm 20$ & $\underline{167}$ & $2417 \pm 41(\underline{2448})$ & $2250 \pm 40(\underline{2281})$ \\
$\Sigma_{b}, \Lambda_{b}^{+}(u d) b$ & $18 \pm 5$ & $220 \pm 50$ & $5754 \pm 68$ & $5534 \pm 118$ \\
& & & & \\
$\Xi_{c}^{+}(u s) c$ & $47 \pm 20$ & $110 \pm 35$ & $2573 \pm 41$ & $2463 \pm 76(\underline{2460})$ \\
$\Xi_{b}^{+}(u s) b$ & $15 \pm 5$ & $150 \pm 35$ & $5904 \pm 68$ & $5754 \pm 93$ \\
& & & & $6916 \pm 139$ \\
$\Xi_{b c}^{+}(b c) u$ & $37 \pm 15$ & $60 \pm 4$ & $6976 \pm 99$ & $7073 \pm 130$ \\
$\Omega_{b c}^{0}(b c) s$ & $27 \pm 10$ & $60 \pm 4$ & $7133 \pm 90$ & \\
\hline
\end{tabular}


TABLE IV: Candidates for Excited Baryons which Only Decay Radiatively to the Ground-State Baryon

\begin{tabular}{lc}
\hline \hline \multicolumn{2}{c}{ Reasonably Certain } \\
\hline$\Xi_{c}^{+\prime}(u s c)$ & $110 \pm 35 \mathrm{MeV}$ \\
$\Omega_{c}^{0 *}(s s c)$ & $42 \pm 15$ \\
$\Xi_{c c}^{+*}(c c d)$ & $56 \pm 20$ \\
$\Omega_{c c}^{+*}(c c s)$ & $42 \pm 15$ \\
$\Omega_{b}^{-*}(b s s)$ & $13 \pm 5$ \\
$\Xi_{b c}^{+\prime}(b c u)$ & $60 \pm 40$ \\
$\Xi_{b c}^{+*}(b c u)$ & $107 \pm 55$ \\
$\Omega_{b c}^{0 \prime}(b c s)$ & $60 \pm 40$ \\
$\Omega_{b c}^{0 *}(b c s)$ & $87 \pm 50$ \\
$\Omega_{c c b}^{+*}(b c c)$ & $4 \pm 2$ \\
$\Xi_{b b}^{-*}(b b d)$ & $18 \pm 5$ \\
$\Omega_{b b}^{-*}(b b s)$ & $13 \pm 5$ \\
$\Omega_{b b c}^{0 *}(b b c)$ & $4 \pm 2$ \\
\hline
\end{tabular}

Marginal

\begin{tabular}{ll}
\hline$\Xi_{c}^{+*}($ usc $)$ & $157 \pm 55$ \\
$\Xi_{b}^{+\prime}$ (bus) & $150 \pm 35$ \\
$\Xi_{b}^{+*}$ (bus) & $165 \pm 35$ \\
\hline
\end{tabular}

